\title{
Effect of the Antiglaucoma Agent JV-GL1 and Related Compounds in the Canine Eye
}

\author{
David F. Woodward, ${ }^{1,2}$ Robert A. Coleman, ${ }^{3}$ Amanda J. Woodrooffe, ${ }^{3}$ \\ Clayton S. Spada, ${ }^{4}$ and Jenny W. Wang ${ }^{2}$
}

\begin{abstract}
Purpose: JV-GL1 is an efficacious, potent, and long-acting antiglaucoma agent, according to studies in ocular normotensive and hypertensive monkeys. As an obligatory step in the drug development process, studies with exaggerated doses and an accelerated dosing schedule for JV-GL1 were performed in a second species (dog). Methods: Intraocular pressure (IOP) was measured by pneumatonometry in conscious Beagle dogs, which remained conscious throughout the study and gently restrained by hand. Pupil diameter was measured with an Optistick. Ocular surface hyperemia was visually assessed and scored according to a 1-3 assessment scale.

Results: JV-GL1, as a $0.01 \%$ eye drop, produced significantly greater reductions in IOP than the original clinical dose of bimatoprost $(0.03 \%)$. JV-GL1 and its free acid enzymatic hydrolysis product PGN 9856, over a $0.01 \%-0.1 \%$ dose range, reduced IOP to $\leq 10 \mathrm{~mm} \mathrm{Hg}$. JV-GL1 and PGN 9856 produced no miosis but a similar degree of ocular surface hyperemia to bimatoprost. Although PGN 9862, a close congener of PGN 9856, was very active as the free acid, esterification essentially abolished its ocular hypotensive activity and ocular surface redness.

Conclusion: JV-GL1 was confirmed as a highly effective and potent ocular hypotensive, exceeding the activity of bimatoprost. A similar degree of ocular surface redness was apparent for both compounds, given as eye drops, but no other effects occurred. Results with PGN 9862 and its isopropyl ester confirmed that PGN 9862isopropyl ester is not bioavailable in the eye and not susceptible to enzymatic hydrolysis in ocular tissues, a first for $\mathrm{C} 1$ ester prodrugs in the eye.
\end{abstract}

Keywords: JV-GL1, dog, intraocular pressure, pupil

\section{Introduction}

$\mathbf{F}$ OLLOWING 2 DECADES of research, the first prostanoid $\mathrm{EP}_{2}$ receptor agonist was recently approved for the clinical treatment of glaucoma. ${ }^{1-3}$ A new second generation compound, JV-GL1, exhibits unprecedented efficacy and duration of action never before seen in an antiglaucoma agent, according to data obtained from ocular normotensive and ocular hypertensive monkeys. ${ }^{3-5}$ There can be, however, a wide species-dependent variation in the pharmacological regulation of ocular surface and anterior segment biology. ${ }^{6}$ The laser induced ocular hypertensive monkey ${ }^{7-9}$ is widely regarded as the most reliable model with respect to clinical translation. In the drug development process, aimed at providing data to drug regulatory authorities to determine the suitability of new compounds for administration to human subjects, information in at least 2 different species is required. This being the case, studies on the ocular pharmacological profile of JV-GL1 and its congener PGN 9862 were repeated in a second species. The dog was selected as the second species for study, as this species was extensively used in the pharmacological characterization of bimatoprost. ${ }^{10}$

The studies herein involved exaggerated doses and dosing intervals. A once-weekly dosing regimen was sufficient for JV-GL1 to reduce intraocular pressure (IOP) in ocular hypertensive monkey eyes to that of ocular normotensive

\footnotetext{
${ }^{1}$ Department of Bioengineering, Imperial College London, South Kensington, United Kingdom.

${ }^{2}$ JeniVision, Inc., Irvine, California, USA.

${ }^{3}$ Asterand Ltd. Royston, Herts, England.

${ }^{4}$ Clayton Spada Visual Communications, Beijing, China.
} 
eyes. ${ }^{5}$ This long-acting regulation of IOP was achieved with a single eye drop of $0.006 \%$ JV-GL $1 .{ }^{5}$ Doses in the $0.01 \%$ $-0.1 \%$ range were dosed daily in these present canine studies, and the free acid enzymatic hydrolysis product of JV-GL $1^{5}$ was also studied. In addition, a direct paired comparison to bimatoprost was performed.

\section{Methods}

\section{Intraocular pressure}

IOP studies in dogs involved pneumatonometry performed on conscious Beagle dogs of both sexes $(10-15 \mathrm{~kg})$. The animals remained conscious throughout the study and were gently restrained by hand. Drug solutions were administered topically to 1 eye as a $25 \mu \mathrm{L}$ volume drop; the other eye received $25 \mu \mathrm{L}$ vehicle $(0.1 \%$ polysorbate $80: 10 \mathrm{mM}$ tromethamine [TRIS]) as a control. All drugs were given once daily. JV-GL1, PGN 9862-isopropyl ester, and their free acid analogs were given to 1 eye, and either vehicle (1\% polysorbate: $10 \mathrm{mM}$ TRIS) or bimatoprost $0.03 \%$ was administered to the contralateral eye. This experimental design allowed a direct paired comparison. IOP was determined just before drug administration and at 2, 4, and $6 \mathrm{~h}$ thereafter on each day of the 5 day study. Drugs were administered once a day, immediately after the first IOP reading. A measure of $0.1 \%$ proparacaine (Ophthetic, Allergan, Irvine, CA) was used for corneal anesthesia during tonometry.

\section{Pupil diameter}

Dog pupil diameter was measured using an Optistick (a mm ruler, which included half-circle references of standard widths) for reference. Gently restraining the dog by hand, pupil diameter was determined by matching a halfcircle to the pupil in normal room light. In dogs with very dark pupils a specialized penlight was used, but only very briefly to avoid pupil constriction. Pupil diameter was measured at the same time as IOP and hyperemia. Like all other dog studies, pupil diameter studies were carried out by Charles River Laboratories (Redfield, AR).

\section{Ocular surface hyperemia}

Ocular Surface Hyperemia was visually assessed and scored according to the following schematic:

\begin{tabular}{lc}
\hline Hyperemia score & Assigned value \\
\hline Trace & 0.5 \\
Mild & 1 \\
Moderate & 2 \\
Severe & 3 \\
\hline
\end{tabular}

Ocular surface hyperemia was also evaluated at the same time points as IOP measurement. It should be noted that untreated dog eyes frequently have a pink/red tone. Thus, values of 0.5 and even 1 are not necessarily out of the normal range. The data are graphically depicted with a nonlinear time (hours) $\mathrm{x}$ axis, to permit clearer visualization of the data at each time point.

The dog IOP studies were performed at Charles River Laboratories and BASi Evansville, Inc. (Mount Vernon, IN). The dogs were randomly assigned to each study, and the individuals who conducted the study were unaware of the drug identities. All studies complied with the ARVO Statement for the Use of Animals in Ophthalmic and Vision Research and were approved by all institutional review boards.

JV-GL1, PGN-9862-isopropyl ester, and their free acid analogs were synthesized at Target Molecules (Southampton, UK).

\section{Results}

The effects of JV-GL1 on beagle dog IOP are shown in Fig. 1. A once-daily $0.01 \%$ dose of JV-GL1 produced profound decreases in IOP that exhibited no fade over the 5-day experimental time course (Fig. 1a). A direct and paired comparison of JV-GL1 $(0.01 \%)$ with bimatoprost $(0.03 \%)$ is provided in Fig. 1b. In this comparison, JV-GL1 was clearly and significantly more effective than bimatoprost in lowering IOP. JV-GL1 could lower IOP to $10 \mathrm{~mm} \mathrm{Hg}$; this was not the case for bimatoprost (Fig. 1b). The effects of graded doses of the enzymatic hydrolysis product of JV-GL1, PGN $9856,{ }^{3}$ are presented in Fig. 2. PGN 9856 exhibited a high degree of ocular hypotensive activity at $0.01 \%$ (Fig. 2a), $0.03 \%$ (Fig. 2b), and $0.1 \%$ (Fig. 2c) doses.

The effects of PGN 9862-isopropyl ester, a close structural analog of JV-GL1, on IOP are shown in Fig. 3a. PGN 9862-isopropyl ester, at a $0.01 \%$ daily dose, showed minimal activity compared to the vehicle treated contralateral eyes. Few statistically significant reductions occurred, and the absolute numerical decreases in IOP were small. The free acid analog PGN 9862, at daily $0.1 \%$ doses, produced substantial decreases in IOP (Fig. 3b), similar to those produced by PGN 9856.

JV-GL1 (Fig. 4a), and its enzymatic hydrolysis product PGN 9856 (Fig. 5a-c), did not cause miosis in the dog eye. Bimatoprost served as a positive control and caused profound pupil constriction in $\operatorname{dogs}^{10}$ (Fig. 4b), to the point where the pupil was sometimes so miniscule that it was difficult to visualize. PGN 9862-isopropyl ester (Fig. 6a) and its free acid analog (Fig. 6b) did not cause pupil constriction.

Ocular surface redness is an unwanted side effect common to all prostanoid based antiglaucoma therapies. For this reason, it was systematically assessed and assigned a rating for JV-GL1, PGN 9862, and their isopropyl esters. Bimatoprost $(0.03 \%)$ was used as a positive control. During the initial $6 \mathrm{~h}$ postdosing on the first day of the experiment, JVGL1 $(0.01 \%)$ produced a greater degree of ocular surface redness compared to bimatoprost (Table 1a). On subsequent days 2-4, JV-GL1 produced more ocular surface redness than bimatoprost only up to the 4-h postdosing time point. After the initial periods of red eye, the redness produced by JV-GL1 consistently decreased to mild. In contrast, the ocular surface redness produced by bimatoprost did not partially resolve during the entire period of the 5 day study, but gradually increased throughout the study (Table 1). Similar results were obtained for JV-GL1 (0.01\%) in a study comparing to vehicle applied to the contralateral eye (Table 1b).

The free acid version of JV-GL1 (PGN 9856) also produced ocular surface hyperemia (Table 2a). Comparing JVGL1 and PGN 9856 at $0.01 \%$ doses given daily for 5 days, PGN 9856 appeared to cause less ocular surface hyperemia. PGN $9856 \quad 0.03 \%$ (Table 2b) and PGN $9856 \quad 0.1 \%$ 

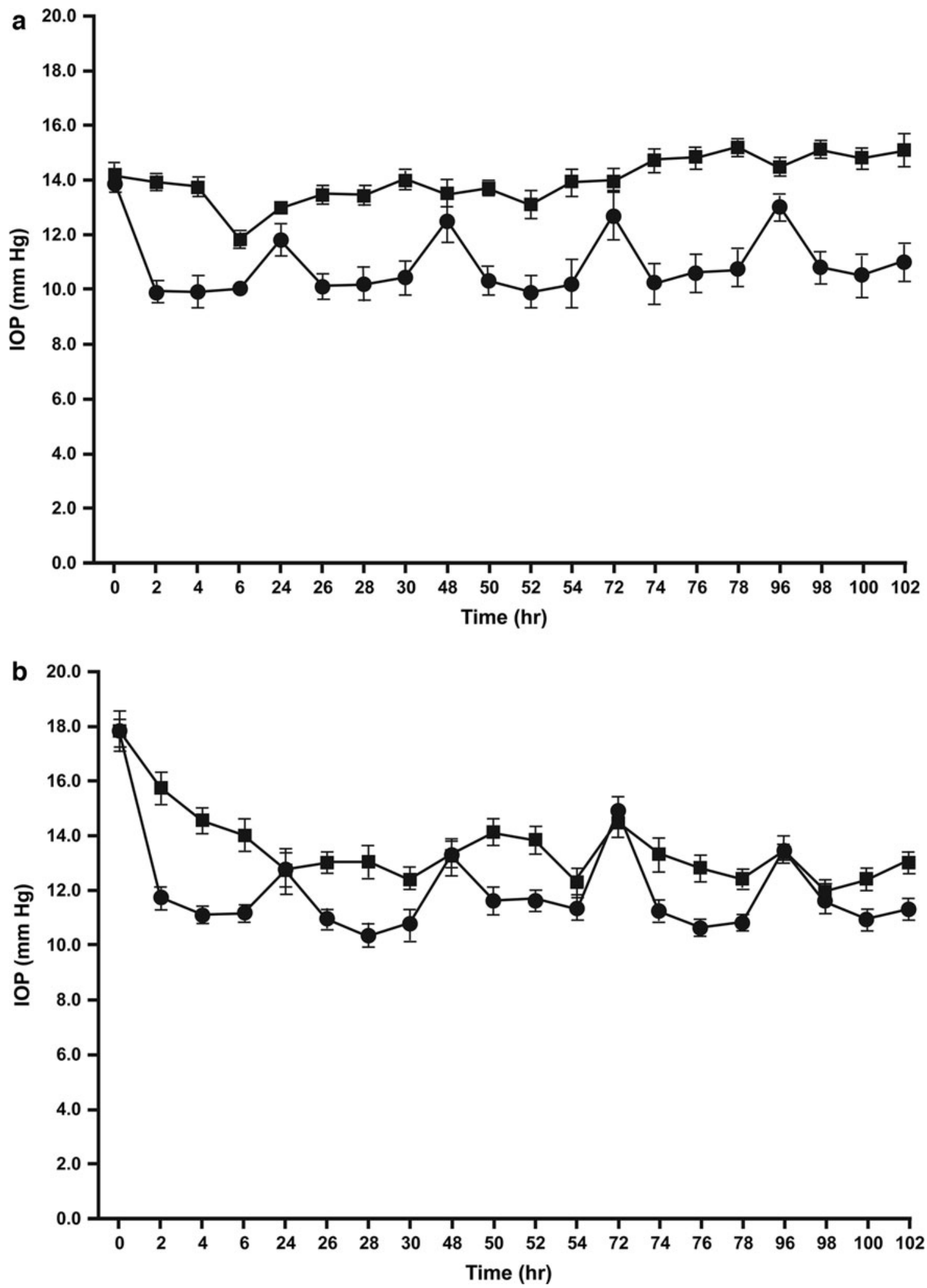

FIG. 1. The effect of JV-GL1 (0.01\%), administered as an eye drop once-daily, on dog IOP. (a) compares the activity of $0.01 \%$ JV-GL1 ( ) with contralaterally applied vehicle $(\boldsymbol{\square})$. Values are mean \pm SEM, $n=6$. Statistically significant differences, $* P<0.05$ and $* * P<0.01$ according to Student's paired $t$-test, were obtained between eyes at the following time points (hours); **2, **4, **6, **26, **28, **30, **50, **52, **54, **74, **76, **78, *96, **98, **100, **102. (b) compares the activity of $0.01 \%$ JV-GL1 ( ) with contralaterally applied $0.03 \%$ bimatoprost ( $\square$ ). Values are mean \pm SEM, $n=6$ per group. Statistically significant differences, ${ }^{*} P<0.05$ and $* * P<0.01$ according to Student's paired $t$-test, were obtained between eyes at the following time points (hours); **2, **4, **6, **26, **28, *30, **50, **52, *74, **76, **78, $* 100, * * 102$. IOP, intraocular pressure; SEM, standard error of the mean. 

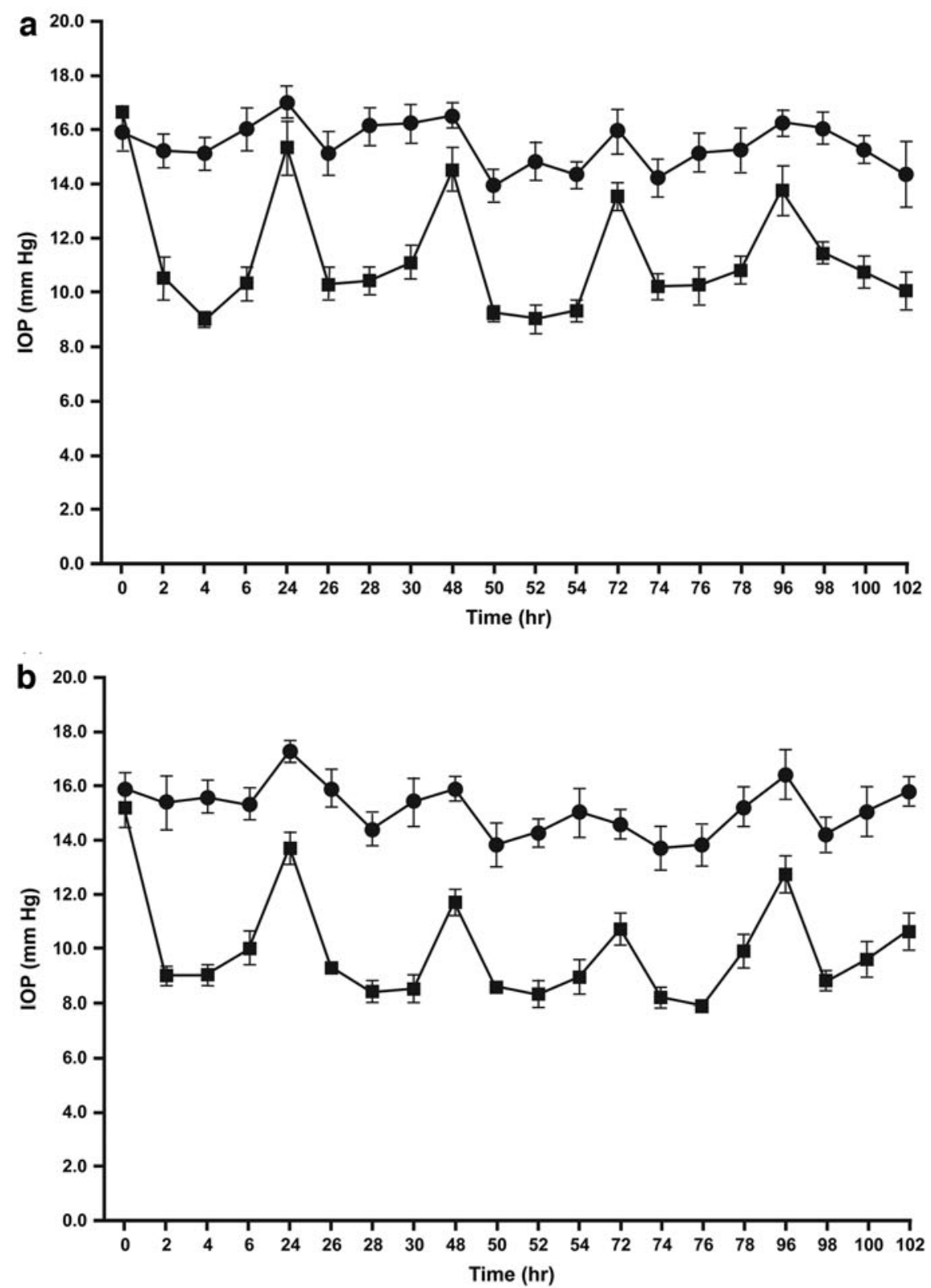

FIG. 2. The effect of graded doses of the free acid analog of JV-GL1, PGN 9856 ( $\boldsymbol{\square}$ ), on dog IOP: (a) $0.01 \%$, (b) $0.03 \%$, (c) $0.1 \%$ compared to vehicle control (O) Values are mean \pm SEM, $n=6$ per group. Statistically significant differences, $* P<0.05$ according to Student's paired $t$-test, were obtained between eyes at the following time points (hours); (a) $0.01 \%$ PGN 9856, *96 (b) $0.03 \%$ PGN 9856, *24, *48, *72, *96 (c) $0.1 \%$ PGN 9856, *48, *72. Statistically significant differences, $* * P<0.01$ according to Student's paired $t$-test, were obtained between eyes at the following time points (hours) and for all doses of PGN 9856; **2, **4, **6, **26, **28, $* * 30, * * 50, * * 52, * * 54, * * 74, * * 76$, $* * 78, * * 100, * * 102$.

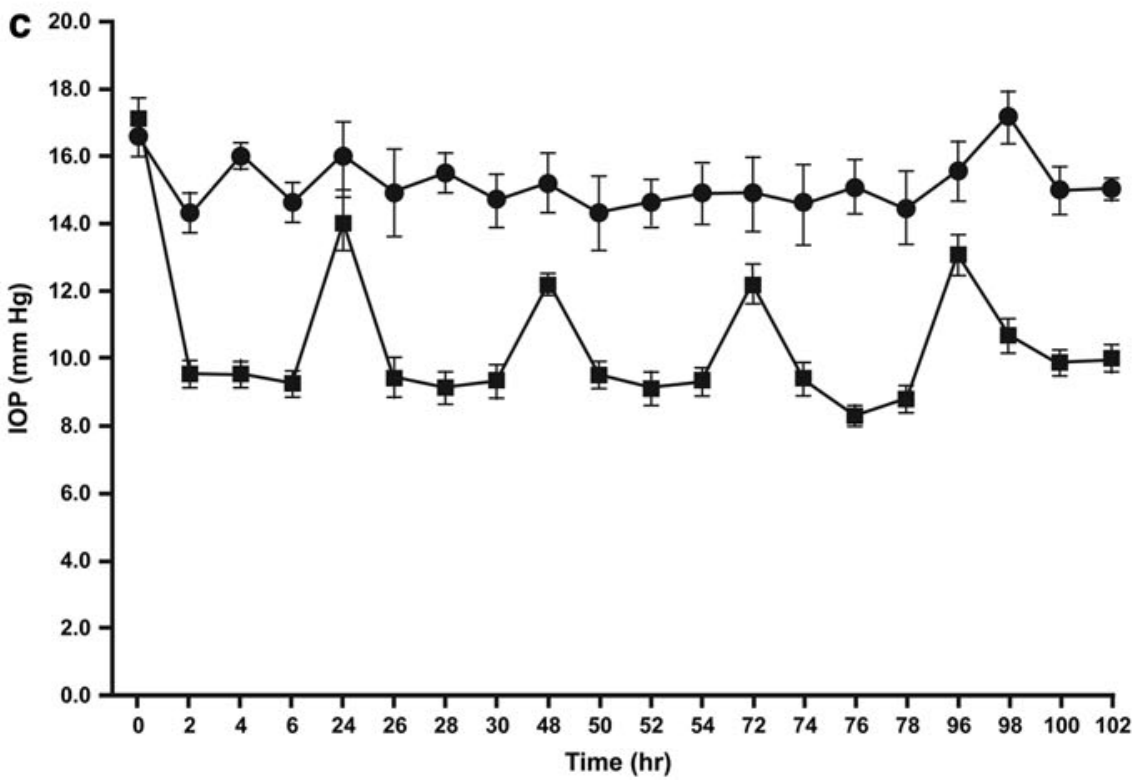



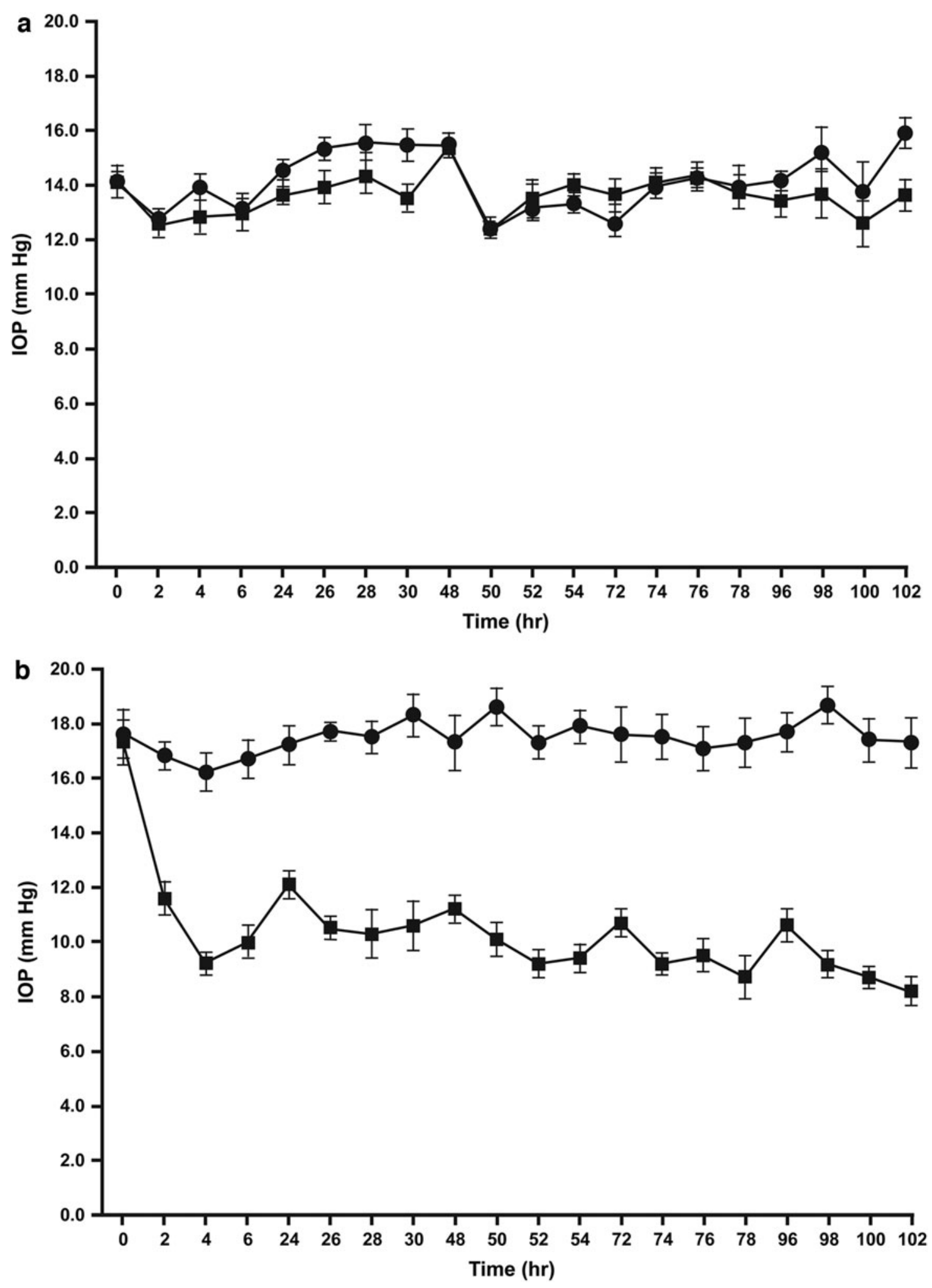

FIG. 3. Effect of (a) PGN 9862-isopropyl ester ( $\mathbf{a}$ ) and (b) PGN 9862 (free acid $\mathbf{0}$ ) on dog intraocular pressure compared to vehicle control ( ). For PGN 9862-isopropyl ester, statistically significant differences, ${ }^{*} P<0.05$ according to Student's paired $t$-test, were obtained between eyes only at 2 time points, 30 and $102 \mathrm{~h}$ (a). For PGN 9862 (b), Statistically significant decreases, ${ }^{* *} P<0.01$ in IOP according to Student's paired $t$-test, were obtained between eyes at all postdosing time points. Values are mean \pm SEM, $n=6$. 

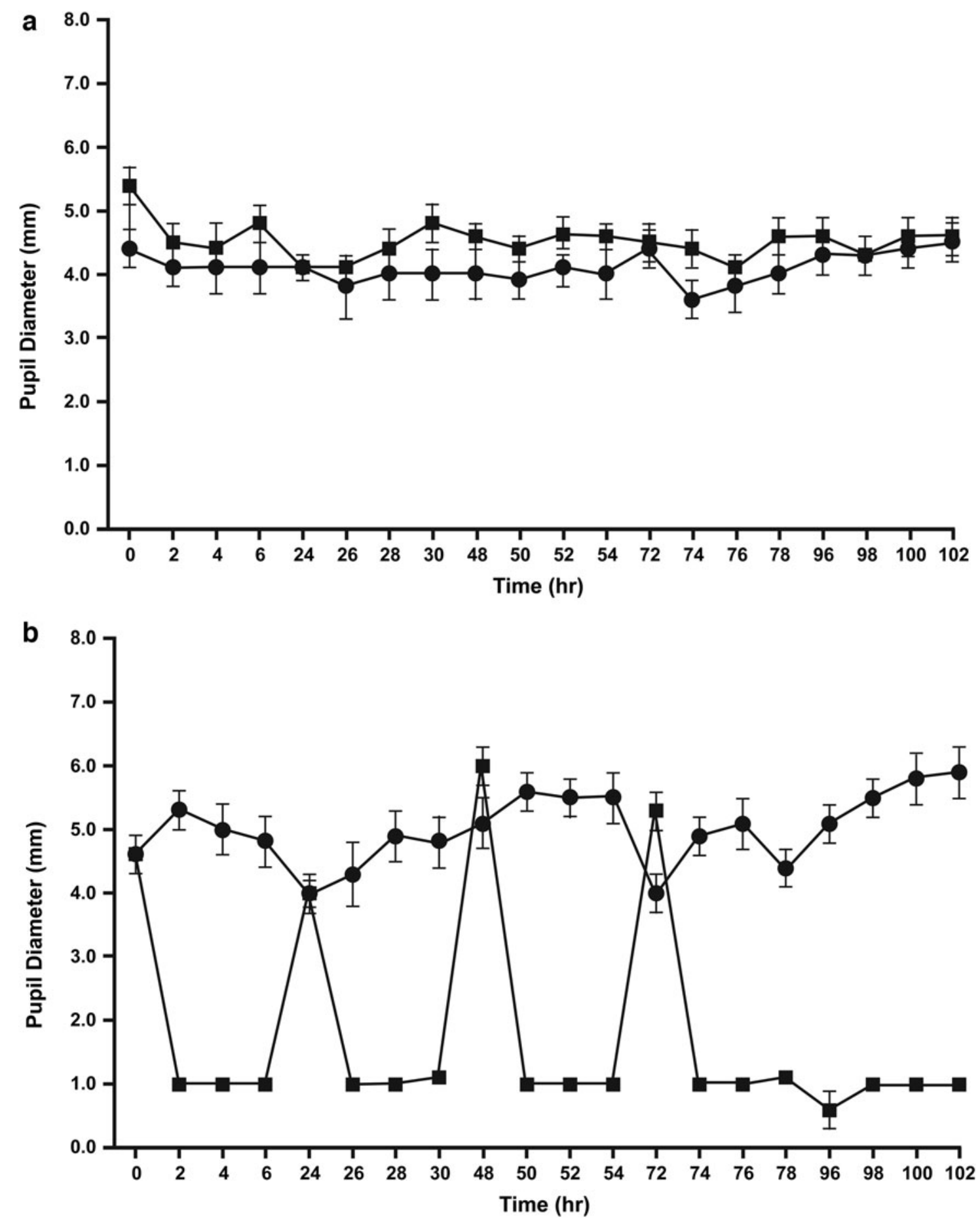

FIG. 4. Comparison of the effect of (a) $0.01 \%$ JV-GL1 ( $\mathbf{a})$ versus vehicle ( $\bigcirc$ ) and (b) $0.01 \%$ JV-GL1 ( $($ ) versus $0.03 \%$ bimatoprost ( $)$ on dog pupil diameter. JV-GL1 produced no significant effect on pupil diameter. In very marked contrast, $0.03 \%$ bimatoprost produced profound miosis that was significantly different $* * P<0.01$ from JV-GL1 at the following time points postdosing (hours); **2, **4, **6, **26, **28, **30, **50,**52,**54,**74, **76, **78, **96, **98, **100, $* * 102 \mathrm{~h}$. Values are mean $\pm \mathrm{SEM}, n=6$.

(Table 2c) produced a much greater degree of ocular surface redness that was frequently in the moderate score range.

The effects of PGN 9862 and PGN 9862-isopropyl ester are compared in Table 3. PGN 9862-isopropyl ester administered once daily for 5 days (Table 3a) caused no ocular surface hyperemia relative to the contralateral eye that received vehicle. During the final day of the study, the assessed ocular surface redness in both eyes inexplicably reached a value of $\sim 1.5$ in this particular experiment. PGN 9862 per se, given once daily at a $0.1 \%$ dose, caused clear ocular surface redness that achieved the moderate range on several occasions (Table 3b). Apart from ocular surface hyperemia, no other ocular side effects were noted for all compounds. 


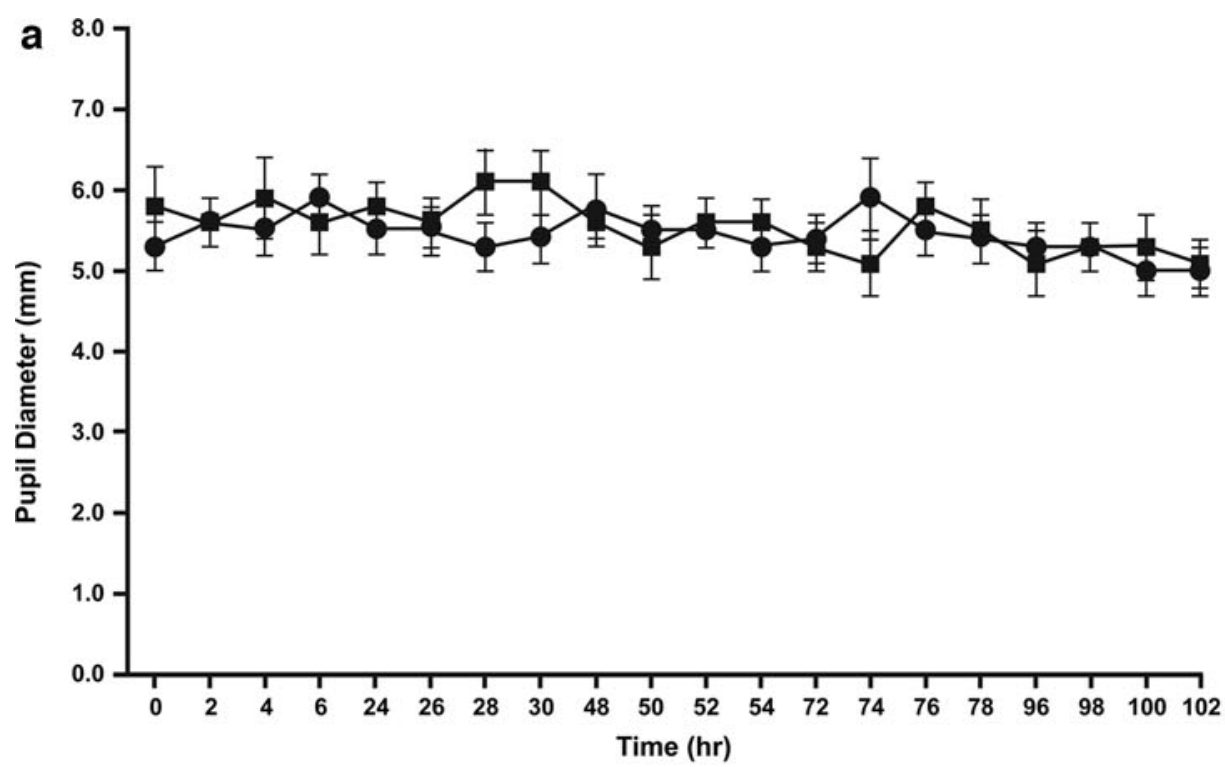

FIG. 5. Effect of (a) $0.01 \%$, (b) $0.03 \%$, (c) $0.1 \%$ PGN 9856 (ם) on dog pupil diameter compared to contralaterally applied vehicle $(\mathbf{O})$. A statistically significant difference in pupil diameter $* P<0.05$ occurred only for the $0.1 \%$ dose of PGN 9856 at the 4 and $6 \mathrm{~h}$ time points $(\mathbf{c})$. Values are mean \pm SEM, $n=6$.
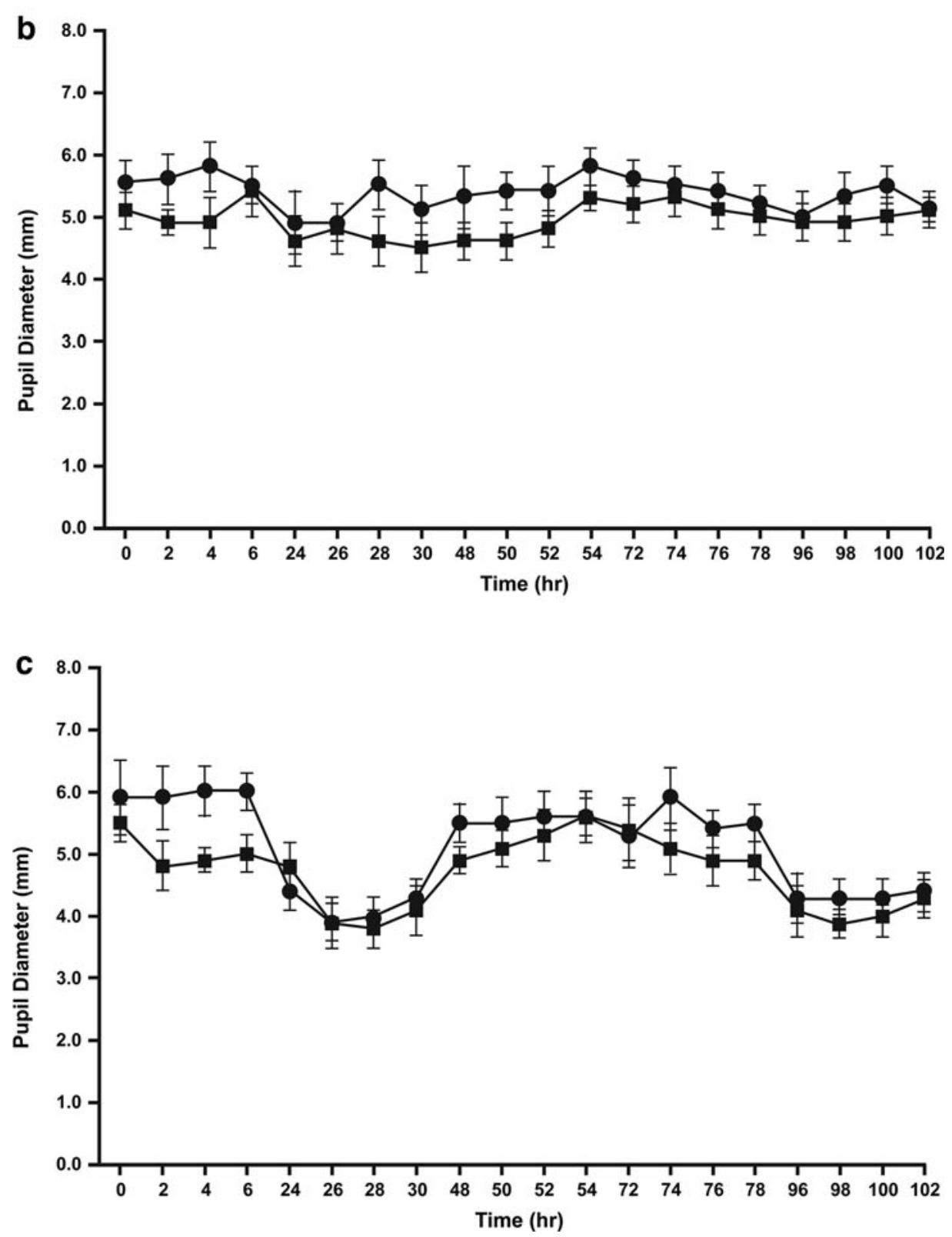

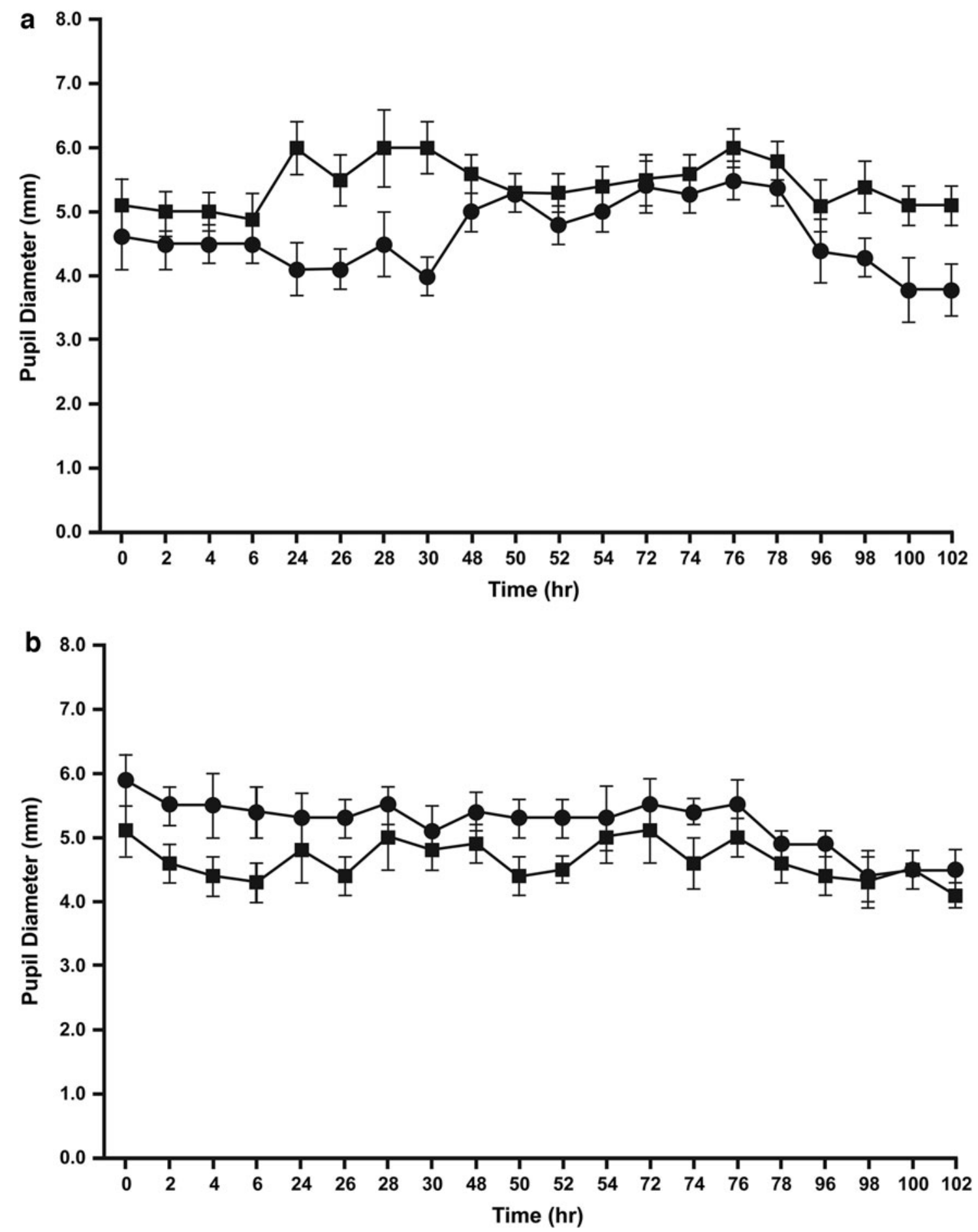

FIG. 6. Effect of (a) $0.01 \%$ PGN-9862-isopropyl ester ( $\boldsymbol{\square})$ compared to vehicle (O) and (b) $0.1 \%$ PGN 9862 ( $\square$ ) compared to vehicle ( ) on dog pupil diameter. PGN 9862-isopropyl ester caused modest pupil dilation at both ends of the experimental time scale (a) and statistical significance was achieved $* P<0.05$ at the $26,28,98$, and $102 \mathrm{~h}$ and $* * P<0.01$ at the 24 and $30 \mathrm{~h}$ time points. PGN 9862 actually seemed to cause modest pupil constriction (b), which was statistically significantly $* P<0.05$ at the $52 \mathrm{~h}$ time point. Values are mean \pm SEM, $n=6$.

\section{Discussion}

JV-GL1 was confirmed as a highly effective ocular hypotensive agent in a second species, the dog. JV-GL1, as the ester and as the free acid product of enzymatic hydrolysis, was capable of reducing dog IOP to $10 \mathrm{~mm} \mathrm{Hg}$ and below.
Similar results were previously obtained in monkeys but at lower doses than those used in these present dog studies. ${ }^{5}$ Moreover, no attempt was made to reproduce the long-acting effects found in the monkey studies. ${ }^{5}$ The dog studies described herein involved exaggerated doses and dosing intervals. Their principal objective was to reveal any unwanted or 


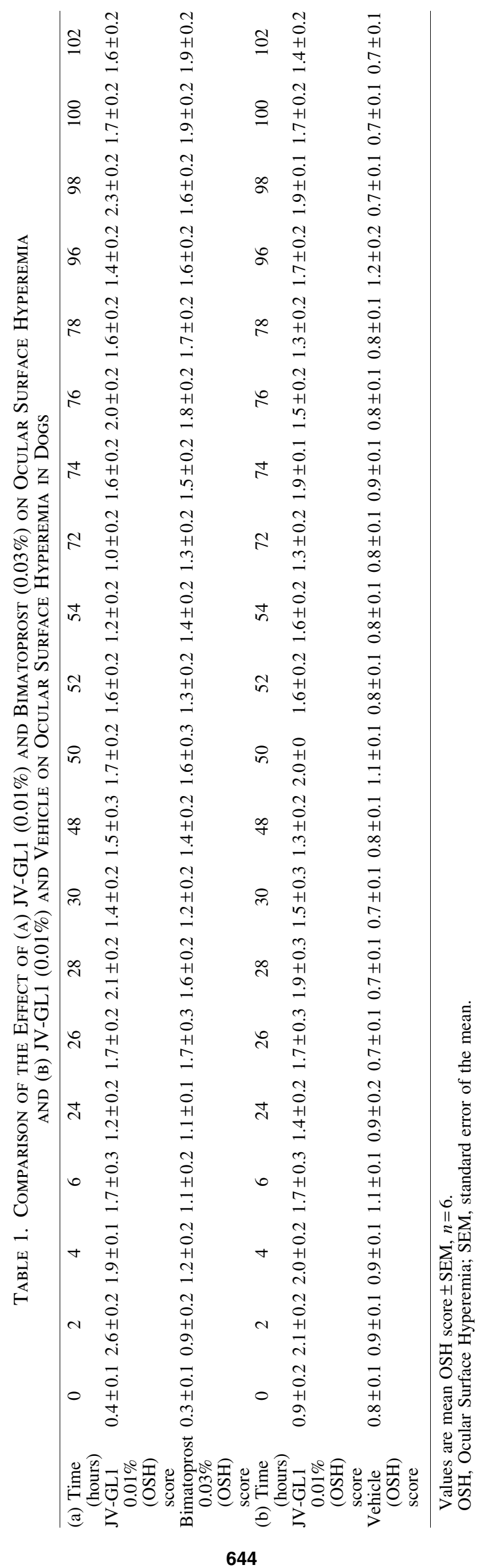




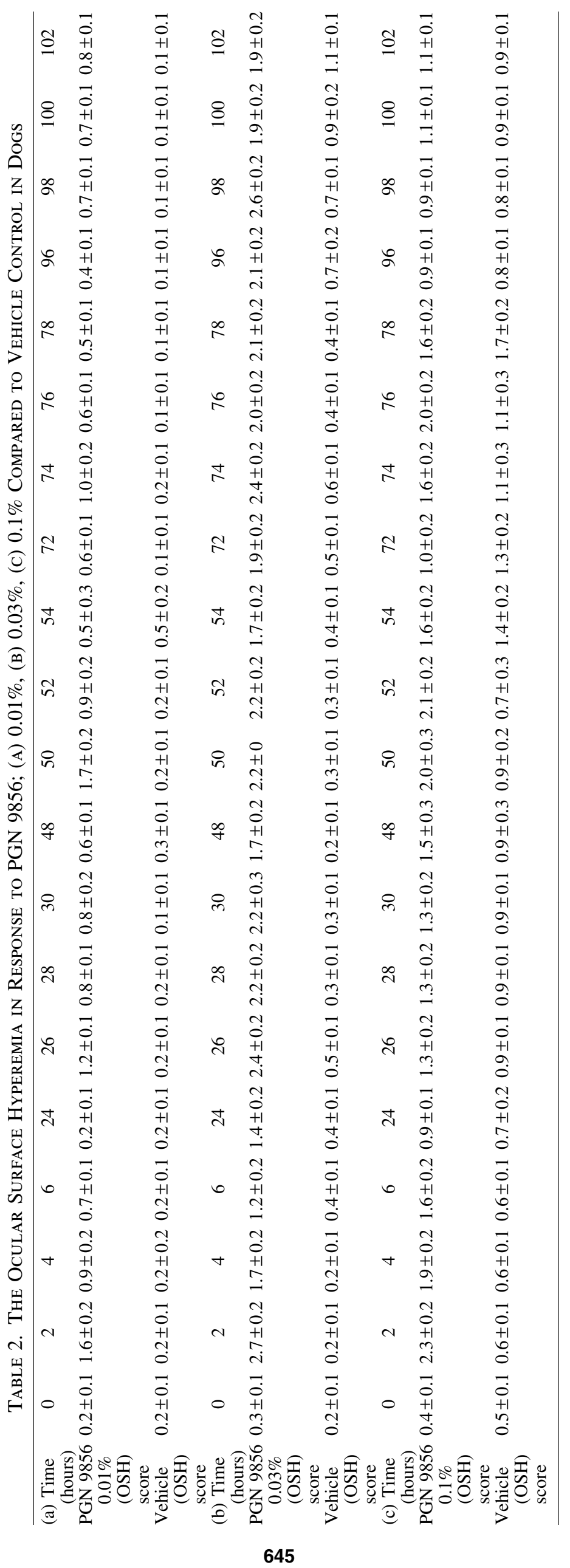




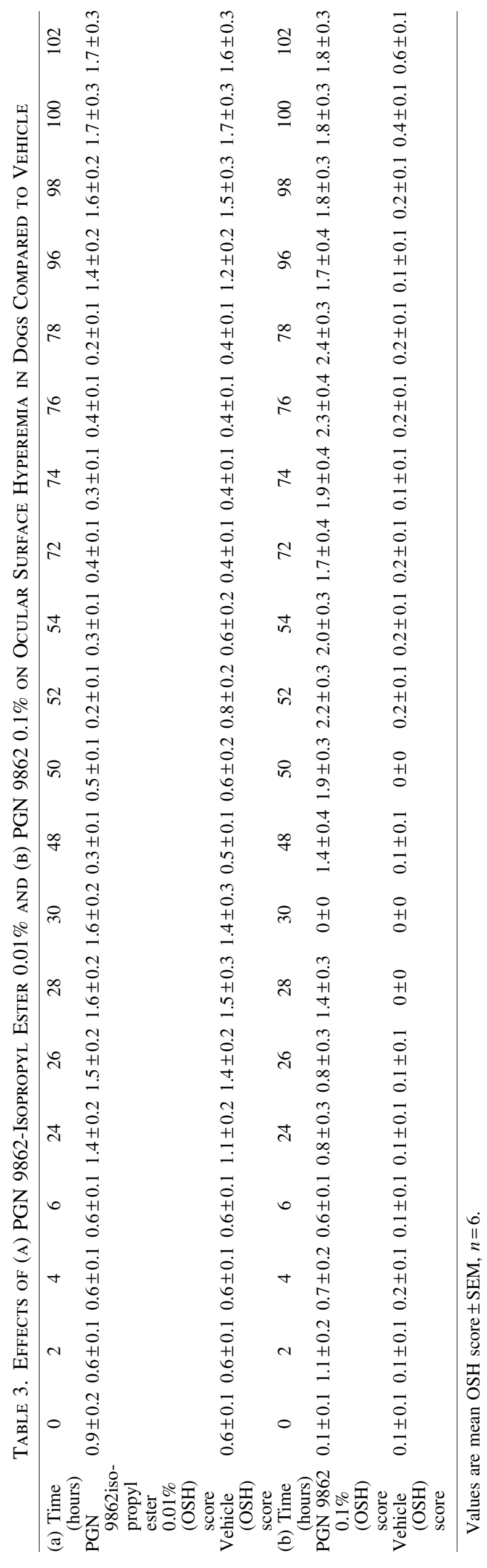


unexpected effects not previously revealed: these could include tachyphylaxis or fade of the ocular hypotensive efficacy, crossover effects in the contralateral eye, excessive ocular surface hyperemia, nociceptive events, tearing, or alteration in pupil diameter. None of these events occurred in association with an exaggerated dosing regimen of JV-GL1.

Antiglaucoma agents that use the cAMP/PKA pathway as an intracellular signal transduction pathway, such as $\beta_{2^{-}}$ adrenoceptor agonists, tend to develop tachyphylaxis. ${ }^{11}$ This results in a rapid loss of ocular hypotensive activity. The free acid product of JV-GL1 enzymatic hydrolysis, PGN 9856, potently increased cAMP levels in human $\mathrm{EP}_{2}$ receptor transfected cells. ${ }^{4}$ Nevertheless, on repeated administration of high doses of JV-GL1, PGN 9856, and PGN 9862 to the eye, no evidence of tachyphylaxis or any fade of ocular hypotensive efficacy was apparent. This means that either once-weekly or once-daily dosing regimens are feasible for JV-GL1. It was also confirmed that the effects of JV-GL1 remained localized to the treated eye and were not the result of systemic transfer to the contralateral eye or the brain. As an example of systemic effects on IOP, the ocular hypotensive effects of brimonidine in monkeys are bilateral, and a central imidazoline receptor has been purported to be involved. ${ }^{12}$

JV-GL1 and all of the compounds appeared to be comfortable when applied to the ocular surface. In a model predictive for ocular surface redness in association with $0.03 \%$ bimatoprost, ${ }^{10} \mathrm{JV}-\mathrm{GL} 1$ at a $0.01 \%$ dose also produced ocular surface hyperemia. This was more pronounced during the first few hours postdosing but was equivalent to bimatoprost later in the experiment. It should be noted, however, that once-daily $0.01 \%$ eye drops is far above the intended clinical dosing regimen for JV-GL1.

Bimatoprost is a potent miotic in dogs. ${ }^{10}$ In these present studies, bimatoprost was highly effective in producing miosis and caused what is described as "pin-point pupil." Repeated doses of bimatoprost also cause a modest degree ( $\sim 15 \%)$ of pupil constriction in monkeys. ${ }^{10} \mathrm{JV}-\mathrm{GL} 1$, PGN 9856, PGN 9862-isopropyl ester, and PGN 9862 had no meaningful effect on pupil diameter, and any significant differences that occurred between paired eyes would likely not have occurred if compared to baseline values.

Previous studies on PGN 9862-isopropyl ester revealed a most unexpected activity profile. It was inactive as an ocular hypotensive despite the close structural identity of PGN 9862 to PGN 9856 and PGN 9862 being essentially equi-active with PGN 9856 as an $\mathrm{EP}_{2}$ agonist. ${ }^{5}$ As in the monkey, PGN 9862-isopropyl ester did not lower IOP in dogs. Moreover, PGN 9862-isopropyl ester did not cause ocular surface hyperemia in dogs. This was particularly surprising because its free acid analog, PGN 9862, clearly caused red eye.

In a previous study on the ocular bioavailability of PGN 9862-isopropyl ester in monkeys, no meaningful penetration into the anterior segment tissues was apparent..$^{5}$ This was most surprising, since the derivatization of prostanoids to the relatively aqueous stable isopropyl ester has been invariably used to improve penetration into the globe. Although present in the conjunctiva, ${ }^{5}$ the same study also revealed that the metabolic conversion of PGN 9862isopropyl ester was much slower compared with JV-GL1 (PGN 9856-isopropyl ester) in the bulbar conjunctiva, the anatomical location of those visible blood vessels on the ocular surface and overlying the sclera. At first hour postdosing, PGN-9862-isopropyl ester was present at
$369.2 \mathrm{ng} / \mathrm{g}$ tissue and the free acid metabolite (PGN 9862) at $21.5 \mathrm{ng} / \mathrm{g}$ tissue; $5.8 \%$ conversion. ${ }^{5}$ In marked contrast, JV-GL1 (PGN 9856-isopropyl ester) at first hour postdosing was present at $10.8 \mathrm{ng} / \mathrm{g}$ tissue and the free acid (PGN 9856) at $179.9 \mathrm{ng} / \mathrm{g}$ tissue; $94 \%$ conversion. ${ }^{5}$ The low concentrations of PGN 9862 in the bulbar conjunctiva would explain the relative absence of ocular surface redness following PGN 9862-isopropyl ester administration to the ocular surface.

Many factors may influence ocular bioavailability and resultant effects outside and within the eye. Nevertheless, since PGN 9862 is clearly at least as active as PGN 9856 on IOP, it follows that differences in activity clearly reside with hydrolytic conversion of the ester moiety, but how? There is not always a correlation between drug lipophilicity and biodisposition in the cornea and conjunctiva. ${ }^{13}$ This essentially correlates with studies in a single animal on alkyl acyl monoesters of ascending size and complexity. ${ }^{14}$ For example, for efficacy on IOP over $6 \mathrm{~h}$ the activity ranking was 15 isobutyryl $=15$-isovaleryl $>15$-acetyl $>15$-pivaloyl and, in a separate series, 9-pivaloyl >9-acetyl >9-isobutyryl. ${ }^{14}$ Although clear separation of ocular hypotensive efficacy was apparent, this was not the case for ocular surface hyperemia, and all animals exhibited clear ocular surface hyperemia at clinically useful doses. ${ }^{14}$

For JV-GL1 and PGN 9862-isopropyl ester, the mechanistic difference between the enzymatic hydrolysis conversion rates resides in the $\mathrm{O}$ atom at position $\mathrm{C} 3$ of JV-GL1 and the 2, 3 double bond of PGN 9862-isopropyl ester. The electronegative $\mathrm{O}$ at $\mathrm{C} 3$ of JV-GL1would render the carbonyl C-1 more electropositive, thereby facilitating enzymatic hydrolysis. This mechanism whereby the $\mathrm{O}$ atom of JV-GL1 withdraws electron density from the carbonyl $\mathrm{O}$ atom of the ester grouping is supported by synthetic chemistry protecting group chemistry where a methoxyacetyl ester cleaves 20 times faster than acetyl. ${ }^{15}$ Other contributory factors that may slow the enzymatic hydrolysis of PGN-9862-isopropyl ester may include less flexibility of the terminal carboxyl chain imparted by the 2, 3 double bond and/or off-target Michael addition reactions sequestering the ester substrate.

One unexpected finding was that JV-GL1 behaved like a typical esterified $\mathrm{EP}_{2}$ agonist ${ }^{2,4}$ in dogs, in that the ocular hypotensive activity appeared to be fading at $24 \mathrm{~h}$ postdosing. The late-onset and long-acting effects of JV-GL1 observed in monkeys ${ }^{4,5}$ were not reproduced in dogs. This may be explained by anatomical differences of the outflow pathways between dogs and monkeys. Unlike monkeys, ${ }^{16}$ dogs lack a Schlemm's canal, ${ }^{17}$ and the canine pathways of aqueous drainage are thereby distinguished from primates. ${ }^{4}$ Reasonable deduction would suggest that Schlemm's canal is the anatomical location that imparts the long-acting effects of JV-GL1 on IOP. ${ }^{5}$

In summary, studies in dogs confirm JV-GL1 as a safe antiglaucoma agent that would maintain undiminished ocular hypotensive activity. Further studies will determine the mechanism(s) underlying the protracted activity of the JVGL1 small molecule which, in primates, imparts effects from a single eye drop that may persist as long as 2 weeks. ${ }^{5}$

\section{Author Disclosure Statement}

D.F.W. and J.W.W. hold a relevant patent and co-own JeniVision Inc. Other coauthors have no proprietary interests. 


\section{Funding Information}

The studies were financially supported by Asterand Ltd. Royston, England.

\section{References}

1. Woodward, D.F., Lawrence, R.A., Fairbairn, C.E., et al. Intraocular pressure effects of selective prostanoid receptor agonists involve different receptor subtypes according to radioligand binding studies. J. Lipid Med. 6: 545-553, 1993.

2. Kirihara, T., Taniguchi, T., Yamamura, K., et al. Pharmacologic characterization of omidenepag isopropyl, a novel selective EP2 receptor agonist, as an ocular hypotensive agent. Invest. Opthalmol. Vis. Sci. 59:145-153, 2018.

3. Woodward, D.F., Wang, J.W., Stamer, W.D., et al. Antiglaucoma $\mathrm{EP}_{2}$ agonists, a long road that led somewhere. $J$. Ocul. Pharmacol. 35:469-474, 2019.

4. Coleman, R.A., Woodrooffe, A.J., Woodward, D.F., et al. Characterisation of the affinity, intrinsic activity and selectivity of a novel prostaglandin $\mathrm{EP}_{2}$ receptor agonist at human prostanoid receptors. Br. J. Pharmacol. 176:687698, 2019.

5. Woodward, D.F., Wang, J.W., Coleman, R.A., et al. A highly effective and ultra-long acting anti-glaucoma drug with a new periorbital delivery method. J. Ocul. Pharmacol. Ther. 35:265-277, 2019.

6. Bito, L.Z. Species differences in the responses of the eye to irritation and trauma: a hypothesis of divergence in ocular defense mechanisms, and the choice of experimental animals for eye research. Exp. Eye. Res. 39:807829, 1984.

7. Wang, R.F., Schumer, R.A., Serle, J.B., and Podos, S.M. A comparison of argon laser and diode laser photocoagulation of the trabecular meshwork to produce the glaucoma monkey model. J. Glaucoma. 7:45-49, 1998.

8. Woodward, D.F., Krauss, A.H.-P., Chen, J., et al. Pharmacological characterization of a novel anti-glaucoma agent, Bimatoprost (AGN 192024). J. Pharmacol. Exp. Ther. 305:772-778, 2003.
9. Impagnatiello, F., Toris, C.B., Batugo, M., et al. Intraocular pressure-lowering activity of NCX 470, a novel nitric oxide-donating bimatoprost in preclinical models. Invest. Ophthalmol. Vis. Sci. 56:6558-6564, 2015.

10. Woodward, D.F., Phelps, R., Krauss, A.H.-P., et al. Bimatoprost $\left(\right.$ Lumigan $\left.^{\circledR}\right)$ ): a synthetic prostamide analog. Cardiovasc. Drug Rev. 22:103-120, 2004.

11. Phipps, T.L., Potter, D.E., and Rowland, J.M. Effects of ibuterol, a beta-2 adrenergic prodrug, on intraocular pressure. J. Ocul. Pharmacol. 2:225-237, 1986.

12. Burke, J., and Schwartz, M. Preclinical evaluation of brimonodine. Surv. Ophthalmol. 41:Suppl 1:S9-S18, 1996.

13. Chen, D.-S., and Tang-Liu, D.D.-S. Ocular penetration and bioconversion of prostaglandin $\mathrm{F}_{2 \alpha}$ prodrugs in rabbit cornea and conjunctiva. J. Pharm. Sci. 86:1180-1186, 1997.

14. Cheng-Bennett, A., Chan, M.F., Chen G., et al. Studies on a novel series of acyl ester prodrugs of prostaglandin $\mathrm{F}_{2 \alpha} . \mathrm{Br}$. J. Opthalmol. 78:560-567, 1994.

15. Reese, C.B., and Stewart, J.C. Methoxyacetyl as a protecting group in ribonucleoside chemistry. Tetrahedron. Lett. 40:4273-4276, 1968.

16. Lütjen-Drecoll, E., Wiendl, H., and Kaufman, P.L. Acute and chronic effects of pilocarpine on monkey outflow tissues. Trans. Am. Ophthalmol. Soc. 96:171-191, 1998.

17. Van Buskirk, E.M. The canine eye: the vessels of aqueous drainage. Invest. Ophthalmol. Vis. Sci. 18:223-230, 1979.

Received: March 25, 2020

Accepted: April 25, 2020

Address correspondence to:

Dr. Jenny W. Wang

JeniVision, Inc.

Suite 2005270 California Avenue Irvine, $C A 92617$

USA

E-mail: wangjenny@jenivision.com 